\title{
A new transferred feature selection algorithm for customer identification
}

\author{
Bing Zhu · Yongge Niu · Jin Xiao · Bart Baesens
}

Received: date / Accepted: date

\begin{abstract}
Class imbalance brings great challenges to feature selection in customer identification and most of current feature selection approaches cannot produce good prediction on the minority class. A number of studies have attempted to solve this issue by using resampling techniques. However, resampling techniques only use the in-domain information and they cannot achieve good performance when the imbalance is caused by the absolute rarity of the minority class. In this paper, we focus on the issue of feature selection with class imbalance caused by absolute rarity. By introducing the idea of transfer learning, we develop a transferred feature selection method based on the group method of data handling neural network (GMDH). The proposed ensemble neural network extracts information of similar customers from related domains to deal with the information scarcity of the minority class in the target domain. Experiments are done on a real-world application from cigarette company. The results indicate that the new method gives better predictive performance than other benchmark feature selection methods, especially the predictive accuracy of minority high-value customers. At the same time, the new algorithm can help to identify important features that distinguish highvalue customers from low-value ones.
\end{abstract}

B. Zhu $\cdot$ Y. Niu( $\left(\varpi_{)} \cdot\right.$ J. Xiao

Business School, Sichuan University, Chengdu 610064, P.R. China

E-mail: niuyongge@gmail.com

B. Zhu

E-mail: zhubing1866@hotmail.com

J. Xiao

E-mail: xiaojin@scu.edu.cn

B. Baesens

Department of Decision Sciences and Information Management, KU Leuven, Leuven 3000, Belgium

E-mail: bart.baesens@kuleuven.be
Keywords Customer identification · Feature selection Class imbalance - Absolute rarity - Transfer learning . Group method of data handling

\section{Introduction}

Customer identification is the first stage of customer relationship management (CRM), which involves finding and targeting the customers who are most profitable to companies [29]. Feature selection is a useful tool in this process, and it will not only solve the "curse of dimensionality" problem in modeling, but also help companies to identify key feature subset that distinguishes high-value customers from low-value ones [24]. Therefore, feature selection has received considerable attentions in customer identification.

The data used for customer identification are often imbalanced. There are usually much less high-value customers (minority class) than low-value ones (majority class). The class imbalance issue brings great challenges to current feature selection methods. Most of them tend to find features that are apt to classify all customers as belonging to the majority class, which results in high overall accuracy but unacceptably low accuracy with respect to the minority class. This would be useless at detecting the minority high-value customers. Some people have attempted to solve this issue by using resampling techniques $[13,40]$. However, resampling methods do not add any new information to the minority class. So they are only useful if enough data are provided for both class, and could fail when the class imbalance is caused by absolute rarity (or absolute imbalance). Absolute rarity refers to the situation where the minority class has a very small sample size [43]. For most CRM applications massive customer databases are available due to the development of information technology, but there are still many situations in which only a small number of data are available. 
For example, sometimes data collection through surveys or experiments is very expensive both in terms of time and economic cost, and firms can only obtain a small sample size of customer data. New services and products are other sources of small data size, where records of customer behavior are scarce, especially records of high-value customers. Hence, it is necessary to develop some feature selection methods that could deal with class imbalance caused by absolute rarity.

In CRM practice, abundant data of similar customers from other domains are often available. For example, similar customer data might have been gathered at an earlier time or on other areas with the same purpose. Clearly, customer data from related domains provide valuable information, especially information of the minority high-value customers. As Japkowicz and Stephenin have shown [19], relatively more information about the minority class benefits the classification modeling, which is then able to distinguish rare minority instances from the majority ones. Thus, these data from the related domain could help to alleviate the problem of class imbalance due to the shortage of the minority samples in the domain of interest (hereafter, referred to as the target domain, and the related domain is referred to as the source domain). However, data distributions in different domains are different. Thus, it is an interesting research topic to find suitable ways to utilize the information in the source domain. Recently, the so-called transfer learning technique has been proposed to exploit source domain data in a principled manner [32].

Transfer learning is an emerging family of machine learning technique. It applies the knowledge learned in related domains to develop efficient models in the target domain [4]. It has been proposed to deal with the situations where data are difficult to collect in the target domain while auxiliary data in the source domain are readily available or relatively easy to collect. Previous research has shown that transfer learning can improve modeling accuracy in many areas such as text mining [11], image analysis [27]. To the best of our knowledge, no reported research has applied transfer learning to solve the class imbalance issue in feature selection.

In this paper, we focus on the issue of feature selection with class imbalance caused by absolute rarity. We try to introduce the idea of transfer learning into feature selection and help to relieve information scarcity of the minority class by integrating the auxiliary data from the source domains. For this purpose, an ensemble group method of data handling neural network (GMDH) is developed. The new method, called transferred feature selection based on GMD$\mathrm{H}$ (TFSG), first creates multiple training datasets incorporating both source and target data via sampling and then uses GMDH neural network to select feature subsets for each training dataset. One advantage of GMDH neural network is that it will automatically select significant variable with minimum expertise of CRM analysts [34]. Finally, feature subsets obtained by the GMDH algorithm are aggregated to obtain the optimal feature subset through a cost-weighted average approach. The empirical study based on customer data from a cigarette company shows that the new algorithm can provide good prediction, especially for the high-value target customers, and it also help the decision-makers to identify key customer characteristics.

The remainder of this paper is organized as follows: In Section 2, we present a brief description of feature selection and class imbalance issue , transfer learning and GMD$\mathrm{H}$ neural network. Section 3 elaborates the proposed new algorithm TFSG. In Section 4, we evaluate the performance of TFSG and compare it with benchmark feature selection methods on a real-world application. Finally, in Section 5, we present the study's conclusions.

\section{Related works}

\subsection{Feature selection and class imbalance}

Feature selection first originated from the field of pattern recognition. In customer relationship management, feature selection not only helps companies to identify key determinants of customer behaviors, but also saves a large amount of computational time and cost. Thus, we have witnessed various applications of feature selection in customer relationship management. For example, Piramuthu [33] presented a new feature selection methodology based on blurring measure for customer financial credit-risk evaluation. Kim et al.[23] proposed a new evolutionary local feature selection algorithm for customer targeting. Tseng and Huang [39] introduced rough set theory (RST) to feature selection for predicting customer purchasing behavior. Huang et al. [17] applied a new multi-objective feature selection approach to churn prediction in telecommunications service field. Chen and $\mathrm{Li}$ [8] utilized SVM classifier combinined with conventional statistical LDA, Decision tree, rough sets and F-score approaches as a feature pre-processing step to discriminate between good and bad customers in credit scoring. Oreskiet al. [31] proposed a heuristic for feature selection process using genetic algorithms. Farquad [12] carried out the support vector machine recursive feature elimination (SVM-RFE) algorithm in the customer churn prediction model. All of these studies shown the benefits of feature selection.

Class imbalance is often encountered in CRM, especially in customer identification. Class imbalance is a very hot topic in the past decade. It is considered to be one of the ten challenging problems in data mining research [45]. Although many solutions for handling class imbalance has been proposed, most of them focus on the classification task. Only several works attempted to tackle this issue for feature selection task. In the study of Van Hulse et al. [40], the influence of resampling techniques on feature selection was 
examined by using five imbalanced microarray expression datasets. Four sampling methods were considered in their work, namely, random undersampling, random oversampling, synthetic minority over-sampling (SMOTE) and Wilson editing. Khoshgoftaar et al. [22] presented a process involving a random undersampling technique for addressing uneven class distribution to select important attributes in software engineering. Gao et al.[13] proposed a new technique, called SelectRUSBoost, which is a form of ensemble learning that incorporates random undersampling into feature selection to alleviate class imbalance. Yin et al.[46] presented a novel feature selection approach based on class decomposition to cope with class imbalance issue. As we can see, most of the attempts do not add any new information to the imbalance data and the model performance could be hindered by the absolute rarity.

\subsection{Transfer learning}

The concept of transfer learning comes from psychology[35], and it illustrates the psychological observation that humans can often benefit from previous learning experiences when learning a new related task. For instance, a good checker player may find it easier to learn playing chess than other people. Thrun[38], Baxter[2] and Caruana [6] introduced the idea into data mining and machine learning field in the mid1990s under the labels of "learning how to learn" , "lifelong learning" and "multi-task learning". At the beginning of this century, Ben-David and Schuller[3] presented the notion of relatedness between learning tasks, which provides theoretical justification for transfer learning. The outline of transfer learning is given in Fig. 1 and a comprehensive survey of transfer learning is provided by Pan and Yang [30].

Instance transfer is the most popular method for transfer learning. It assumes that some instances in the source domains are helpful for training the target domain model, while others could do harm to target learning task. Instancetransfer uses re-weighting or resampling techniques to select those useful source domain instances. Many instance-based transfer learning approaches for classification task have already been proposed. For example, Dai et al. [9] proposed a boosting-style method to utilize the old data in related domains. Jiang and Zhai [20] presented an instance-weighting approach to exploit auxiliary information from the source domain based on the difference between the conditional probabilities. Recently, Kamishima et al.[21] modified the bagging ensemble algorithm to develop an algorithm called transfer bagging (TrBagg). TrBagg augments the model performance in the target domain by aggregating the base model$s$ that are built on datasets incorporating the source domain data. The proposed approach TFSG is also an instance-based transfer learning method for feature selection. It tries to in-

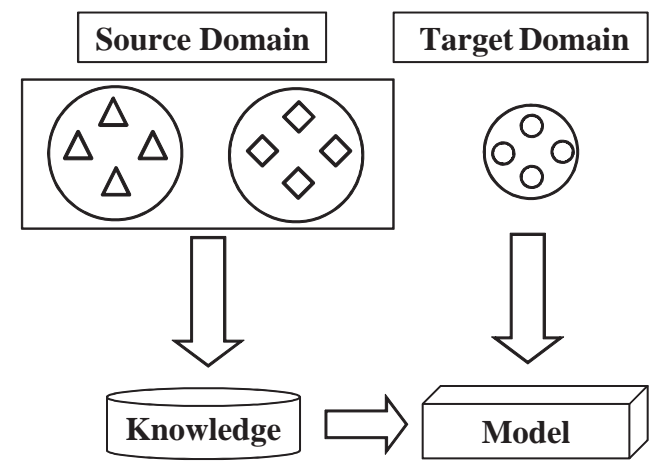

Fig. 1 Outline of transfer learning

corporate information from useful source domain instances to tackle the absolute rarity in the target domain.

\subsection{GMDH neural network}

The GMDH neural network is an inductive modeling method that constructs a hierarchical (multi-layered) network structure to identify complex input-output functional relationship from data. This method was first developed by Ivakhnenko [18] as a multivariate analysis method for complex systems modeling and identification in the 1960s. In the 2000s, Lemke and Muller [26] further developed the GMDH algorithm into the self-organizing data mining algorithm. Since then, GMDH has been successfully applied to many fields such as chemistry [14], engineering [34] and economics [41].

The modeling process of GMDH is analogous to natural evolution of wheat. It is a self-organizing process based on synthesizing models of increasing complexity and selecting of the best solution according to external criterion. The GMDH first produces some simple elementary models (network neurons) by reference functions and uses them as initial input models at the start of modeling process. After generating a large number of competitive models from the initial input models (inheritance), the algorithm selects certain more optimal intermediate models (selection) such that a large number of new competitive models are generated by these intermediate models. Such procedures of inheritance and selection are repeated until an optimal complex model is created. According to the optimal complexity theory, as the model complexity increases, the value of external criterion usually decreases first and then reaches a minimum. It 
later starts to increase again because of model overfitting. The GMDH algorithm will stop when the external criterion reaches its minimum, and an optimal complex model is obtained [28].

One advantage of the GMDH neural network is that it can automatically select input variables. The modeling process of GMDH is iterative. Starting from simple elementary network neurons produced by reference functions, it generates many intermediate models with different variables. The models that yield better external criteria are selected because they contain inputs that have better ability to explain or predict the output. The input variables from the selected models are used to construct more complex models in subsequen$\mathrm{t}$ layers. As the modeling process proceeds, the number of variables in model candidates increases. The procedure will stop when the external criterion reaches its minimum and the optimal complex model with the most effective input variable set is created. In this way, the algorithm can automatically determine the key input variables and exclude the irrelevant ones. Some people have successfully used GMDH for feature selection . For example, El-Alfy and Abdel-Aal [10] presented a technique based on GMDH to identify the most effective content features for classifying emails.

\section{Methodology}

\subsection{Problem definition}

Suppose that there is a dataset in the target domain that is composed of $m$ customer instances $D^{T}=\left\{\left(\mathbf{x}_{i}^{T}, y_{i}^{T}\right)\right\}_{i=1}^{m}$. $\mathbf{x}_{i}^{T} \in X$ is an instance in the $d$-dimensional feature space $X=\left\{f_{1}, \ldots, f_{d}\right\}$, and it describes customers characteristics. $y_{i}^{T}$ is the label of customer class. In this paper, we assume that there are only two types of customers $y_{i}^{T} \in\{0,1\}$ , where $y_{i}^{T}=1$ denotes high-value customer and $y_{i}^{T}=0$ represents low-value customer. The goal of feature selection is to find a feature subset $E=\left\{f_{1}^{\prime}, \ldots, f_{k}^{\prime}\right\} \subset X, k<d$ that can distinguish high-value customers from low-value ones.

In customer identification, firms often face the situation of class imbalance. We define $P^{T} \subset D^{T}$ as the set of highvalue customer instances that belong to the minority class , and $N^{T} \subset D^{T}$ as the set of low-value customer instances belonging to the majority class. Class imbalance refers to the situation in which $\left|P^{T}\right| \ll\left|N^{T}\right|$. When class imbalance is brought by the relatively small size of the minority class $P^{T}$, we call it absolute rarity.

We assume there are $n$ similar customer instances in the source domain, $D^{S} \in\left\{\left(\mathbf{x}_{i}^{S}, y_{i}^{S}\right)\right\}_{i=1}^{n}$, where the feature space $X=\left\{f_{1}, \ldots, f_{d}\right\}$ is the same as in the target domain. The question now is how we can use customer data $D^{T}$ in the target domain to identify a feature subset $E$ that captures the key customer characteristics in the target domain with the help of auxiliary customer data $D^{S}$ from source domain.

\subsection{Transferred feature selection based on GMDH (TFSG)}

We aim to solve the issue of absolute imbalance that is encountered in feature selection and propose a novel instancebased transfer learning technique TFSG. The main idea of TFSG is to import the minority class instances from source domain to augment the information of the minority class in the target domain by using an ensemble GMDH neural network. The algorithm flowchart is shown in Fig. 2.

The TFSG algorithm first uses bootstrap sampling on minority instances of source domain and undersampling on the majority instances of the target domain. Then it combines them with minority instances of target domain to produce several training datasets. After that, the GMDH is used on each training dataset to obtain candidate feature subsets and finally different feature subsets obtained by GMDH are aggregated with a cost-weighted average approach to obtain an optimal feature ranking. There are three aspects of TFSG that should be stressed. First of all, the minority class dataset $P^{S}$ from source domain contains both useful and useless instances, so we use the cost-weighted average to eliminate the interference of useless data. Specifically, cost-weighted accuracy of each base model is computed on the original target training dataset $D^{T}$. If the value of the cost-weighted accuracy(CWA) is high, then we consider that most of the source domain instances that have been used to train these base models are useful for predicting the customer class in the target domain, and these base models will be assigned larger weights. At the same time, smaller weights are assigned to those base models that have low values of CWA. The final feature ranking score is determined by the weight average. Second, we use GMDH to build base model, because it can select feature subset with little expertise. People only need to determine the number of model candidate selected in each layer, which is very easy task for nearly all the problems. Third, one advantage of ensemble feature selection [1] is stability. Previous research has shown that feature selection is not stable on imbalanced datasets [42] and so we adopt ensemble of feature selection to make the results more stable. The main steps of the algorithm are as follows:

Step 1: Employ bootstrap sampling on $P^{S}$ to obtain dataset $\hat{P}_{i}^{S}$

Step 2: Use random undersampling on $N^{T}$ to obtain $\hat{N}_{i}^{T}$, where $\left|\hat{N}_{i}^{T}\right|=\left|\hat{P}_{i}^{S}\right|+\left|P_{i}^{T}\right|$

Step 3: Combine $\hat{P}_{i}^{S}, P_{i}^{T}$ and $\hat{N}_{i}^{T}$ to obtain a training dataset $D_{i}=\hat{P}_{i}^{S} \cup P_{i}^{T} \cup \hat{N}_{i}^{T}$, and use GMDH to build a base model and obtain the feature subset $E_{i}$;

Step 4: Calculate the value of cost weighted accuracy(CWA) on the original target dataset $D^{T}$ for each base model built by GMDH as follows:

$$
C W A=\left(C_{N} * T N R+C_{P} * T P R\right) /\left(C_{N}+C_{P}\right)
$$




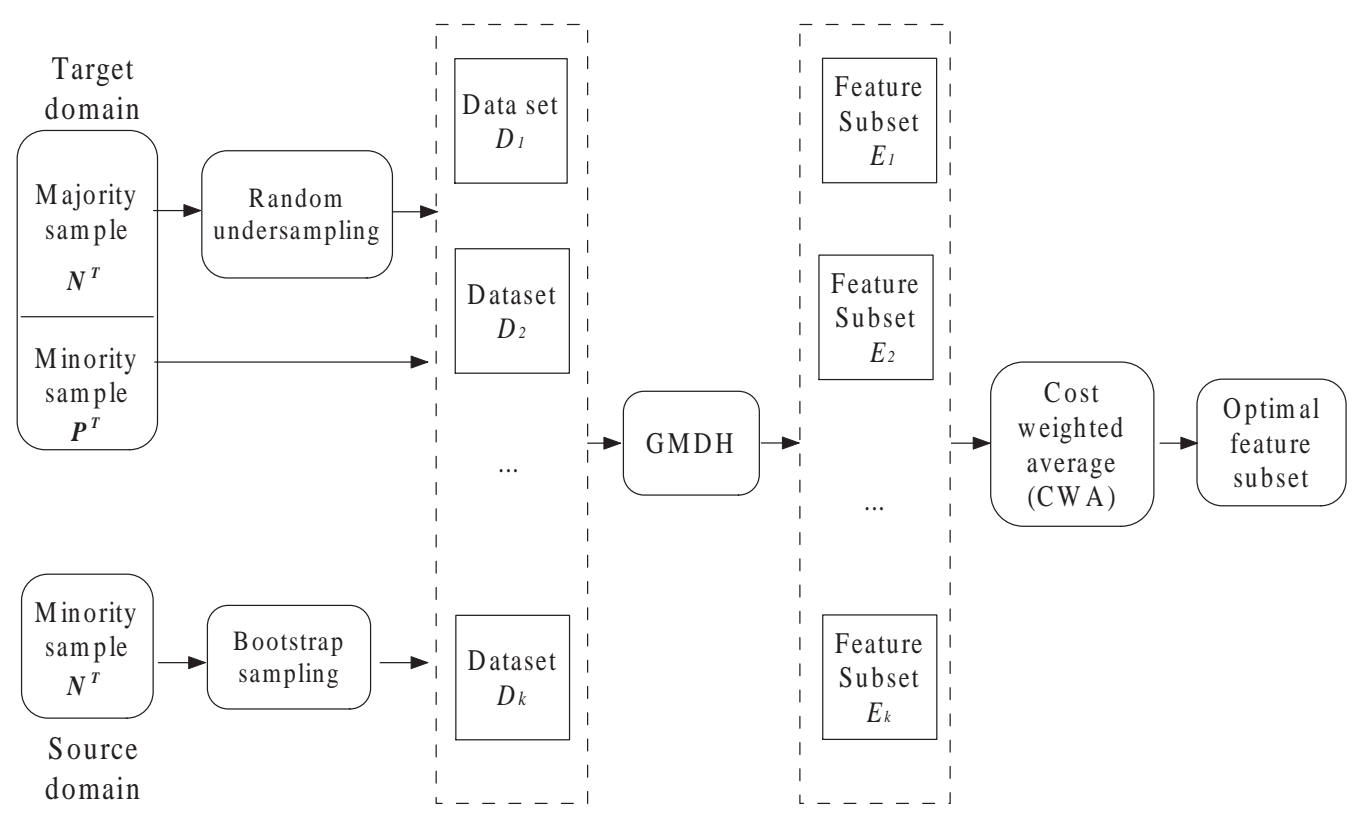

Fig. 2 Flowchart of the TFSG algorithm

where $C_{N}$ and $C_{P}$ are the misclassification cost of the majority and minority class, respectively. $T N R$ and $T P R$ are the true negative rate and true positive rate, respectively;

Step 5: Calculate the feature ranking score $q_{i j}$ for each variable $f_{j}$ according to the value of CWA as follows:

$q_{i j}=\left\{\begin{array}{rll}C W A & \text { if } f_{j} \in E_{i} \\ 0 & \text { if } f_{j} \in E_{i}\end{array} \quad(j=1, \ldots, d)\right.$

Step 6: Repeat Step 2 to Step 5 to obtain $k$ feature subset$\mathrm{s}\left\{E_{1}, E_{2}, E_{3}, \ldots, E_{k}\right\}$ and corresponding ranking scores;

Step 7: Use the following formula to calculate the final aggregated score $Q_{j}$ for each feature:

$Q_{j}=\sum_{i=1}^{k} q_{i j}, j=1, \ldots, d$

In the TFSG algorithm, the following GMDH algorithm is used to construct base models in step 3:

Step 1: Divide dataset $D_{i}$ into two disjoint subsets of the same size $D_{i}=B \cup C$;

Step 2: Combine input variables in pairs $\left(f_{i}, f_{j}\right), 1 \leq i, j \leq d$ and generate model candidates from each combination by using the following quadratic polynomial:

$y=c_{0}+c_{1} f_{i}+c_{2} f_{j}+c_{3} f_{i} \cdot f_{j}+c_{4} f_{i}^{2}+c_{5} f_{j}^{2}$

where $c_{0}, c_{1}, \ldots, c_{5}$ are parameters to be estimated by ordinary least square (OLS) method (see Fig. 3).

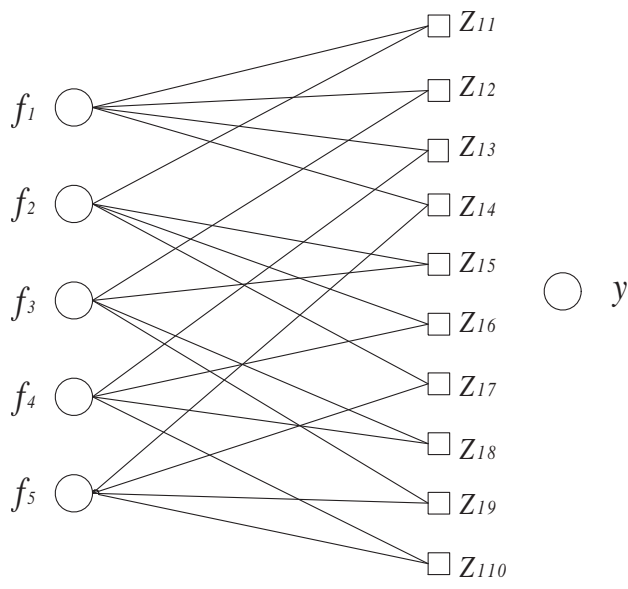

Fig. 3 Generation of candidate models in the first layer

Step 3: Evaluate the external criterion of each model by using symmetric regularity criterion (SRC) as follows:

$$
S R C=\left(\sum_{i \in B}\left(y_{i}-\hat{y}_{i}^{C}\right)^{2}+\sum_{i \in C}\left(y_{i}-\hat{y}_{i}^{B}\right)^{2}\right)
$$

where $y_{i}$ is the actual output, and $\hat{y}_{i}^{B}$ and $\hat{y}_{i}^{C}$ are the estimated outputs of the models that are constructed on dataset $B$ and dataset $C$. Record the minimum of the external criterion $R_{l}$ from current layer;

Step 4: Select $F_{l}$ best models with lower criterion values, and their outputs $z_{l t}$ are employed as new input variables for the second layer of the GMDH network(see Fig. 4). 


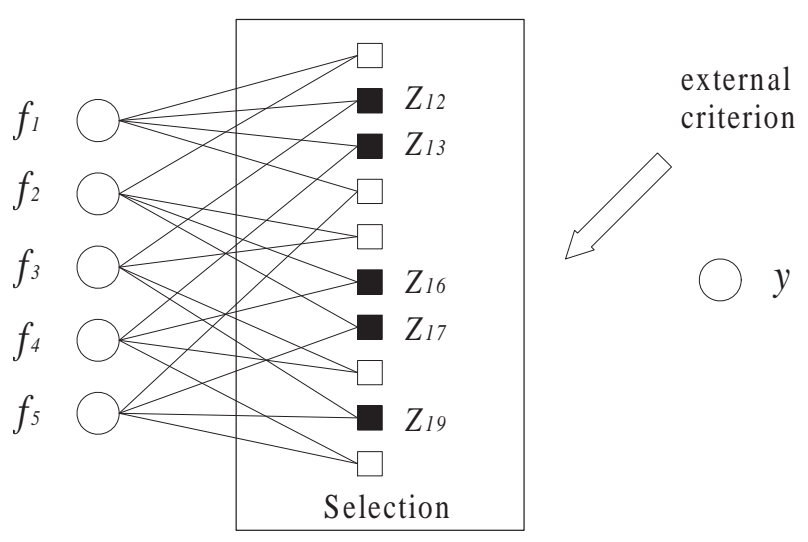

selededcandidamodes notsedededcandidamodas

Fig. 4 Selection of candidate models

Step 5: Repeat Steps 2 to Step 4 to produce model candidates of the second layer, the third layer, and so on, until the lowest value of external criterion at the current layer $R_{l}$ is greater than that in the previous layer. The model with the minimum external criterion at the $l-1$ layer is then selected as the final model. Fig. 5 provides an example where the optimal complex model is obtained at the third layer.

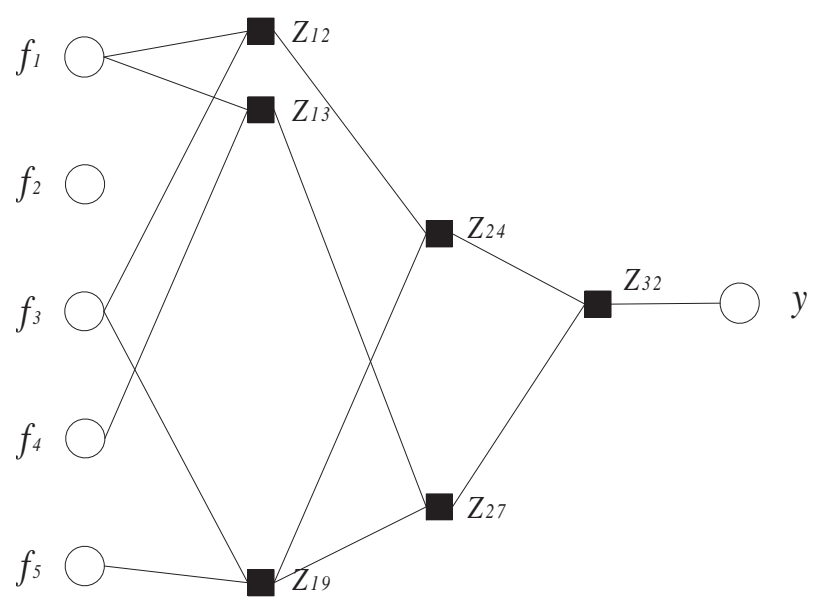

Fig. 5 Generation of the optimal complex model

\section{Experiments}

In this section, we will evaluate the effectiveness of the new method through experiments on a real-world application.

\subsection{Experimental setting}

The data were provided by a cigarette company in Sichuan Province of China. The company intended to launch a new product in the mid-priced cigarette market. Therefore, the managers hoped to understand the drivers of customer behaviors in this market segment, and questionnaire surveys were conducted in Sichuan province in July 2010. The customers were required to provide socio-demographic background, perceptions or attitudes toward products and behavioral information. According to amount of smoking, the customers were divided into heavy (more than 15 cigarettes per day) and mild smokers (less than 15 per day). The heavy smokers are high-value customers. The company obtained a sample with 540 respondents from city SL (target domain), which is a typical dataset with absolute imbalance. There are only 138 heavy smokers, which not only has a smal1 size but is also outnumbered by mild smokers (302 respondents). Fortunately, a similar survey was conducted in city JY (source domain) several months ago, which yielded 420 respondents with 144 heavy smokers. After eliminating variables with too many missing values, we obtained 57 variables from the survey data. Obviously, it not convenient to describe the customers and develop marketing program with all of the 57 variables. Thus, the procedure of feature selection is required.

To justify the effectiveness of the proposed method, five feature selection methods are used as benchmarks: Relief$\mathrm{F}$, information gain (IG), sequential backforward selection (SBS), genetic search (GS) and SVM-RFE. ReliefF and IG are filter methods. ReliefF is an extension of the original Relief algorithm [25]. Previous research has shown that it has good performance regardless of the specific data [5]. IG is an information theoretic method that measures the information gain of the class variable when the attribute variable is given. SBS and GS are wrapper methods. SBS starts from a full set and sequentially removes the feature that results in the smallest decrease in the value of the objective function. The genetic search performs the feature search by using the well-known genetic algorithm. SVM-RFE is an embedded method, and it performs feature selection by iteratively training a SVM classifier using linear kernel with the current set of features and removing the least important feature indicated by SVM [15].

In our study, the target data $\left(D^{T}\right)$ from city SL was randomly divided into a training sample with $70 \%$ of the observations, and a test sample with $30 \%$ of the observations. The data division was performed in a stratified manner to ensure that the proportions of heavy smokers were the same in both training dataset and testing dataset. All methods were applied on the training set to select the feature subsets. For each selected feature subset, a multilayered feedforward neutral network was trained on the selected fea- 
tures, and then tested on the corresponding test set. We used multilayered feedforward neural network as the classifier because it is a widely used method in customer classification. In the two wrapper methods (SBS and GS), the multilayered feedforward neural network was also used as learning scheme. To avoid bias, the above procedures were repeated 30 times, and the average results are reported. Four metrics were used to assess experimental results: accuracy, area under the receiver operating characteristic curve (AUC), sensitivity, and specificity. All of the four metrics give us more insights into model performance[16] in class imbalance setting. After discussion with experts in the cigarette industry, we set the misclassification cost to $C_{P}=1$ and $C_{N}=4$ because the average profit from heavy smokers is usually four times that of mild smokers in the middle-price cigarette market.

In the experiments, we investigated the performance of benchmark feature selection methods in different scenarios and compared TFSG with them. Specifically, we attempt to answer the following three questions:

(1)How does TFSG perform in comparison with the benchmark methods that are trained on the original target data? (2)Will resampling methods have comparable performance as TFSG in addressing class imbalance? (3)Is there any performance difference between TFSG and the benchmark methods trained on the dataset formed by the direct combination of target and source data? To answer these questions, we considered three scenarios in which benchmark methods are built on different datasets. In the first scenario, we only selected the features with benchmark methods on the original target training data $D^{T}$. In the second scenari$\mathrm{o}$, the well known oversampling method synthetic minority oversampling technique (SMOTE) [7] was used on the target training data $D^{T}$ to generate artificial minority instances and get balanced datasets. SMOTE is a powerful method that has shown a great deal of success in various application$\mathrm{s}$ and is still one of the state-of-the-art resampling method. In the third scenario, we combined the minority class data $P^{S}$ from the source domain with the target data $D^{T}$ without any adjustment, and trained models on the combined dataset $D^{T} \cup P^{S}$. All of the benchmark methods were implemented by using the Weka data mining tool with default settings [44]. The TFSG method was programmed by us in matlab.

\subsection{Experimental results}

In this subsection, we will discuss the experimental results to evaluate the effectiveness of the new algorithm. Table 1 through Table 3 report the results of TFSG and benchmark methods in the three experimental scenarios. The best values of each metric are highlighted in bold. We will analyze experimental results in the three above-mentioned scenarios respectively.

\subsubsection{Comparison with benchmark methods on original target data}

We first check the performance of benchmark methods build on the original target data. Table 1 shows the experimental results in this scenario. As Table 1 has shown, each benchmark method gives high accuracy and low sensitivity. The sensitivity values of five benchmark methods are smaller than 0.5 , and IG even has a zero value. This finding indicates that the benchmark methods are affected significantly by uneven class distribution, they cannot help to identify the minority target customers effectively. Meanwhile, TFSG performs well not only in terms of AUC and accuracy, but also in terms of sensitivity. TFSG ranks first with respect to AUC and sensitivity and the superiority is indicated by the corrected resampled $t$-test. In addition, its accuracy ranks in the second place. Thus, we can conclude that TFSG has better performance than the benchmark methods which are significantly impacted by the class imbalance.

Table 1 Experimental results on original target data

\begin{tabular}{lllll}
\hline Method & AUC & Sensitivity & Specificity & Accuracy \\
\hline ReliefF & 0.6454 & 0.4556 & 0.7183 & 0.6510 \\
IG & 0.6982 & 0.0 & $\mathbf{1 . 0}$ & $\mathbf{0 . 7 4 4 0}$ \\
SBS & 0.6759 & 0.2730 & 0.8904 & 0.7323 \\
GS & 0.6713 & 0.1821 & 0.8722 & 0.6955 \\
SVM-RFE & 0.7091 & 0.4554 & 0.8217 & 0.7279 \\
TFSG & $\mathbf{0 . 7 2 1 4}^{*}$ & $\mathbf{0 . 6 3 6 7}^{*}$ & $0.7753^{*}$ & 0.7398 \\
\hline
\end{tabular}

* Statistically significant difference between TFSG algorithm and best benchmarks method at the 5\% significance level

\subsubsection{Comparison with resampling solutions}

Next, we will analyze experimental results using SMOTE sampling technique. Table 2 presents the result in that scenario. We can see from Table 2 that the usage of SMOTE helps benchmark methods improve their ability of identifying the minority class, and degree of improvement is different for different methods. For example, the value of sensitivity increases sharply from 0.0 to 0.6363 for IG after SMOTE sampling, while ReflieF only has a slight increase (from 0.4556 and 0.5089). However, we can find that accuracy and sensitivity of the benchmark methods after using SMOTE sampling can not catch up with TFSG. TFSG performs the best in terms of sensitivity, and its AUC value ranks in second just behind SVM-REF. In terms of accuracy, it also takes the second place. The analysis shows that resampling can alleviate the influence of class imbalance on the benchmark methods, but TFSG has better performance than the benchmark methods with resampling solutions, especially accuracy on the minority class. 
Table 2 Experimental results on target data using SMOTE sampling

\begin{tabular}{lllll}
\hline Method & AUC & Sensitivity & Specificity & Accuracy \\
\hline ReliefF & 0.6434 & 0.5089 & 0.7049 & 0.6547 \\
IG & 0.6643 & 0.6363 & 0.6904 & 0.6765 \\
SBS & 0.7212 & 0.5456 & $\mathbf{0 . 8 4 1 6}$ & $\mathbf{0 . 7 6 5 8}$ \\
GS & 0.6998 & 0.4732 & 0.8061 & 0.7208 \\
SVM-RFE & $\mathbf{0 . 7 2 4 6}$ & 0.5552 & 0.8113 & 0.7457 \\
TFSG & 0.7214 & $\mathbf{0 . 6 3 6 7}$ & $0.7753 *$ & 0.7547 \\
\hline
\end{tabular}

* Statistically significant difference between TFSG algorithm and best benchmarks method at the $5 \%$ significance level

\subsubsection{Comparison with benchmark methods on combined dataset}

We also compare the performance of benchmark methods on the dataset that is formed by direct combination of source and target data. The experimental results are given in Table 3. As the table has shown, the introduction of minority instances from the source data has a mixed influence on benchmark methods. Some benchmark methods improve sensitivity to some extent. For example, the sensitivity of SBS increases from 0.2730 to 0.3649 . On the other hand, some methods perform worse after the source data are introduced, such as SVM-REF which decreases from 0.4554 to 0.3644 . So simple combination is inappropriate because the source and target domain have different distributions. Meanwhile, TFSG makes full use of the information from the source domain more properly. It is ranked in the first position in both AUC and sensitivity, such better performance is also confirmed by the $t$-test. With respect to the specificity and accuracy, it also takes the second place. The experimental results in the third scenario show that TFSG outperforms the benchmark methods trained on the dataset that was formed by direct combination.

Table 3 Experimental results on combined dataset

\begin{tabular}{lllll}
\hline Method & AUC & Sensitivity & Specificity & Accuracy \\
\hline ReliefF & 0.6658 & 0.4673 & 0.7417 & 0.6714 \\
IG & 0.6212 & 0.3121 & $\mathbf{0 . 9 2 3 4}$ & $\mathbf{0 . 7 6 9 4}$ \\
SBS & 0.6323 & 0.3649 & 0.7696 & 0.7255 \\
GS & 0.6755 & 0.5449 & 0.7952 & 0.7311 \\
SVM-RFE & 0.6921 & 0.3644 & 0.7473 & 0.6939 \\
TFSG & $\mathbf{0 . 7 2 1 4}^{*}$ & $\mathbf{0 . 6 3 6 7}^{*}$ & $0.7753{ }^{*}$ & 0.7547 \\
\hline
\end{tabular}

${ }^{*}$ Statistically significant difference between TFSG algorithm and best benchmarks method at the $5 \%$ significance level

\subsubsection{Model interpretability}

Now we come to look at the model interpretability. Fig. 6 shows the 11 features that are selected by TFSG. The values of relative importance are calculated from the original feature ranking scores using a linear transformation, where the largest value will be transformed into 100 , and the smallest value will be transformed to 0 . We will check the performance of the 11 features one-by-one to see how they differentiate heavy smokers from mild smokers through statistical test. Because the 11 selected variables are categorical, twoway contingency tables are used to evaluate whether statistical relationships exist between each selected variable and customer type $y$. The contingency table is a useful tool to analyze the relation between two or more categorical variables [36]. The results are shown in Table 4, where the second column presents the values of the $\chi^{2}$ statistics, the third column reports degrees of freedom (DF), and the fourth column gives the $p$-values.

From Table 4, we can see that nine out of the eleven selected variables yield $p$-values that are smaller than 0.1 based on the $\chi^{2}$ test. The only two exceptions are Acceptability level of arts performing in promotion and Family income, which have $p$-values that are close to 0.1 . The results show that the null hypothesis that there are no significant relations between the selected variables and customer type is rejected at the $10 \%$ significance level. In other words, there is a significant relationship between the selected variables and customer type in the mid-priced cigarette market. In other words, all of the selected variables distinguish the heavy and mild smokers effectively and they can be considered to be the key drivers of customer behaviors in the mid-priced cigarette market.

In summary, the experimental results give us some conclusions to the three research questions presented in section 4.1: TFSG has better predictive performance than both original benchmark methods and benchmark methods with resampling solutions. It also makes full use of auxiliary source data given the distribution difference, thus is superior to benchmark methods trained on the dataset formed by the direct combination of target and source data. In addition, TFSG algorithm results in good model interpretability, the variables selected by it presents important features that differentiate different customers.

\section{Conclusions}

In customer identification the distribution of low-value and high-value customers is often imbalanced. In this paper, we focus on the issue of class imbalance in feature selection caused by absolute imbalance. A new transferred feature selection algorithm based on the GMDH neural network is proposed to deal with this issue. Based on the idea of transfer learning, the proposed method TFSG helps to select a useful feature subset on the target imbalanced dataset by introducing customer information in related domains. We tested the proposed algorithm on a real-world application to analyze 


\section{Relative Importance}

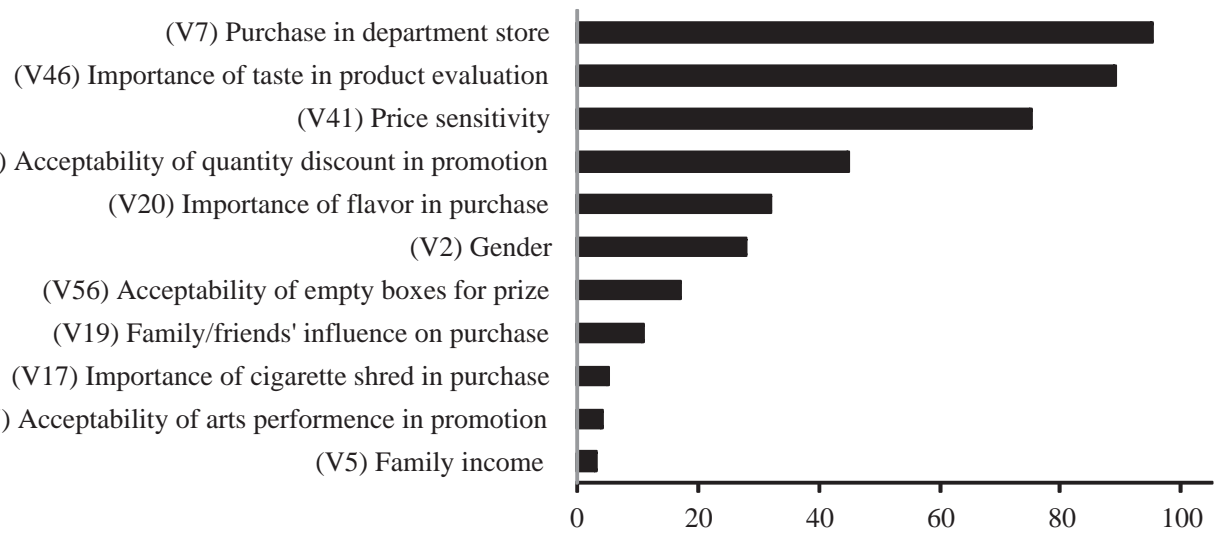

Fig. 6 Relative importance of selected variables

Table 4 Experimental results of contingency table

\begin{tabular}{llll}
\hline Variable & Value & df & $p$-value \\
\hline Purchase in department store & 6.687 & 1 & $<0.01$ \\
Importance level of taste in product evaluation & 7.630 & 3 & 0.0543 \\
Price sensitivity & 5.542 & 2 & 0.0626 \\
Acceptability level of quantity discount in promotion & 9.943 & 5 & 0.0768 \\
Importance of flavor in purchase & 8.04 & 3 & 0.0453 \\
Gender & 3.50 & 1 & 0.0614 \\
Acceptability level of empty boxes for prize & 12.22 & 5 & 0.0320 \\
Family/friend's influence on purchase & 6.414 & 3 & 0.0931 \\
Importance of cigarette in purchase & 6.624 & 3 & 0.0849 \\
Acceptability level of arts performing in promotion & 8.264 & 5 & 0.1422 \\
Family income & 4.203 & 2 & 0.1222 \\
\hline
\end{tabular}

customer characteristics in the cigarette market. It has been demonstrated that the new method TFSG can identify minority high-value customers more effectively than existing feature selection methods. This approach also exhibits better performance than the resampling technique, which is the state-of-the-art method to tackle class imbalance in practice. In addition, the proposed technique can also identify key features that could differentiate high-value and low-value customers with good interpretation. The new algorithm provides a new tool for customer relationship management.

\section{Acknowledgments}

This work is supported by Natural Science Foundation of China (Grant No. 71401115 and 71471124), MOE (Ministry of Education in China) Youth Project of Humanities and Social Sciences (Grant No. 13YJC630249), Specialized
Research Fund for the Doctoral Program of Higher Education of the Ministry of Education of China (Grant No. 20120181120074), and Foundation of Sichuan University (skqy201507).

\section{Appendix}

See Table 5

\section{References}

1. Awada W, Khoshgoftaar TM, Dittman D, Wald R, Napolitano A (2012) A review of the stability of feature selection techniques for bioinformatics data. In: Procceding of 13th IEEE International Conference on Information Reuse and Integration, pp 356-363

2. Baxter J(1997). A Bayesian/information theoretic model of learning to learn via multiple task sampling. Machine Learning 28(1) 7-39 
3. Ben-David S, Schuller R(2003) Exploiting task relatedness for multiple task learning. In: Proceedings 16th Annual Conference on Computational Learning Theory, Washington, DC, USA

4. Bengio Y, Courville A, Vincent P(2013) Representation learning: A review and new perspectives. IEEE Transactions on Pattern Analysis and Machine Intelligence 35(8): 1798-1828

5. Bolon-Canedo V, Sanchez-Marono N, Alonso-Betanzos A(2013) A review of feature selection methods on synthetic data. Knowledge and Information Systems 34(3): 483-519

6. Caruana R(1997) Multitask learning. Machine Learning 28: 41-75

7. Chawla NV, Bowye, KW, Hall LO, Kegelmeyer WP(2002) SMOTE: syntheticminority over-sampling technique. Journal of Artificial Intelligence Research 16(3): 321-357

8. Chen FL, Li FC(2010) Combination of feature selection approaches with SVM in credit scoring. Expert Systems with Applications 37(7): 4902-4909

9. Dai W, Yang Q, Xue G, Yu R(2007) Boosting for transfer learning. In: Proceeding of the 24th International Conference on Machine Learning, ACM Press, pp 193-200

10. El-Alfy EMAbdel-Aal RE (2011) Using GMDH-based networks for improved spam detection and email feature analysis. Applied Soft Computing 11(1): 477-488

11. Faisal A, Gillberg J, Leen G, Peltonen J(2013) Transfer learning using a nonparametric sparse topic model. Neurocomputing 112: 124-137

12. Farquad M, Ravi V, Raju S(2014) Churn prediction using comprehensible support vector machine: An analytical CRM application. Applied Soft Computing 19: 31-40

13. Gao K, Khoshgoftaar TM, Napolitano A(2012) A hybrid approach to coping with high dimensionality and class imbalance for software defect prediction. In: Proceeding of the 11th International Conference on Machine Learning and Applications, pp 281-288

14. Ghanadzadeh H, Ganji M, Fallahi S(2012) Mathematical model of liquid-liquid equilibrium for a ternary system using the GMDHtype neural network and genetic algorithm. Applied Mathematical Modelling 36(9): 4096-4105

15. Guyon I, Weston J, Barnhill S, Vapnik V (2002) Gene selection for cancer classification using support vector machines. Machine Learning 46(1-3):389-422

16. He H, Garcia E(2009) Learning from imbalanced data. IEEE Transactions on Knowledge and Data Engineering 21(9): 12631284

17. Huang B, Buckley B, Kechadi TM(2010) Multi-objective feature selection by using NSGA-II for customer churn prediction in telecommunications. Expert Systems with Applications 37(5): 3638-3646

18. Ivakhnenko AG(1968) The group method of data handling-a rival of the method of stochastic approximation. Soviet Automat. Contr. 1-3: $43-55$

19. Japkowicz N, Stephen S (2002) The class imbalance problem: a systematic study. Intell. Data Anal. 6(5): 429-450

20. Jiang J, Zhai C(2007) Instance weighting for domain adaptation in NLP. In: Proceedings of the 45th Ann. Meeting of the Assoc. for Computational Linguistics, pp 264-271

21. Kamishima T, Hamasaki M, Akaho S(2009) TrBagg: A simple transfer learning method and its application to personalization in collaborative tagging. In: Proceeding of Ninth IEEE International Conference on Data Mining, pp 219-228

22. Khoshgoftaar TM. Gao K, Seliya N(2010) Attribute selection and imbalanced data: problems in software defect prediction. In: Proceeding of International Conference on Tools with Artificial Intelligence, pp 137-144

23. Kim Y, Street W, Russell G, Menczer F(2005) Customer targeting: a neural network approach guided by genetic algorithms. Management Science 51(2): 264-276

24. Kim Y (2006) Toward a successful CRM: variable selection, sampling, and ensemble. Decision Support Systems 41(2): 542-553
25. Kononenkom I(1994) Estimating attributes: analysis and extensions of RELIEF. In: Proceedings of the European conference on machine learning, pp 171-182

26. Lemke F, Mueller J(2003) Self-organising data mining. Systems Analysis Modelling Simulation 43(2): 231-240

27. Luo Y, Liu T, Tao D, Xu C(2014) Decomposition-based transfer distance metric learning for image classification. IEEE Trans Image Process. 23(9): 3789-801

28. Mueller JA, Lemke F(2000) Self-organizing Sata Mining: An Intelligent Approach to Extract Knowledge from data. Libri Books, Berlin, Hamburg

29. Ngai E, Xiu L, Chau D (2009) Application of data mining techniques in customer relationship management: A literature review and classification . Expert Systems with Applications 36: 25922602

30. Pan S, Yang Q (2010) A survey on transfer learning. IEEE Transactions on Knowledge and Data Engineering 22(10):1345-1359

31. Oreski S, Oreski G (2014) Genetic algorithm-based heuristic for feature selection in credit risk assessment. Expert Systems with Applications 41(4): 2052-2064

32. Pan S, Yang Q (2010) A survey on transfer learning. IEEE Transactions on Knowledge and Data Engineering 22(10): 1345-1359

33. Piramuthu S(1999) Feature selection for financial credit-risk evaluation decisions. INFORMS Journal on Computing 11(3): 258266

34. Sheikholeslami M, Sheykholeslami F, Khoshhal S, Mola-Abasia H, Ganji D, Rokni H (2014) Effect of magnetic field on Cu-water nanofluid heat transfer using GMDH-type neural network. Neural Computing \& Applications 25:171C178

35. Skinner BF (1953) Science and Human Behavior. CollilerMacmillian

36. Smith SM, Albaum GS (2005) Fundamentals of Marketing Research. Thousand Oaks, CA: Sage

37. Stepashko VS, Yurachkovskiy YP (1986) The present state of the theory of the group method of data handling. Soviet Journal of Automation and Information Sciences c/c of Avtomatika 19(4): 36-46

38. Thrun $\mathrm{S}(1996)$ Is learning the $\mathrm{N}$-th thing any easier than learning the first?. In: Proceedings of NIPS-96, pp 640-646

39. Tseng TL, Huang CC(2007) Rough set-based approach to feature selection in customer relationship management. Omega 35(4): 365-383

40. Van Hulse J, Khoshgoftaar TM, Napolitano A, Wald R(2009) Feature selection with high-dimensional imbalanced data. In: Proceeding of the 2009 IEEE International Conference on Data Mining Workshops, pp 507-514

41. Venkatesh K, Ravi V, Prinzie A, Van den Poel D(2014) Cash demand forecasting in ATMs by clustering and neural networks. European Journal of Operational Research 232(2): 383-392

42. Wang H, Khoshgoftaar TM, Napolitano A(2012) An empirical study on the stability of feature selection for imbalanced software engineering data. In: Proceeding of 11th International Conference on Machine Learning and Applications (ICMLA), pp 317-323

43. Weiss GM (2004) Mining with rarity: a unifying framework. ACM Sigkdd Explorations Newsletter 6(1): 7-19

44. Witten IH, Frank E (2005) Data Mining Practical Machine Learning Tools and Techniques. Morgan Kaufmann, San Francisco

45. Yang Q, Wu X. (2006) 10 challenging problems in data mining research. International Journal of Information Technology Decision Making 5(4): 597-604

46. Yin L, Ge Y, Xiao K, Wang X, Quan X (2013) Feature selection for high-dimensional imbalanced data. Neurocomputing 105(1) 311 
Table 5 Overview of explanatory variables

\begin{tabular}{|c|c|c|c|c|}
\hline Category & ID & Description & Type & Range \\
\hline \multirow{5}{*}{$\begin{array}{l}\text { Socio- } \\
\text { demographic } \\
\text { predictor }\end{array}$} & V1 & - Age & Continuous & {$[16,70]$} \\
\hline & $\mathrm{V} 2$ & - Gender & Binary & $\{0,1\}$ \\
\hline & V3 & - Educational level & Ordinal & $\{1,2,3\}$ \\
\hline & V4 & - Personal income & Ordinal & $\{1,2,3\}$ \\
\hline & V5 & - Family income & Ordinal & $\{1,2,3\}$ \\
\hline \multirow{5}{*}{$\begin{array}{l}\text { Behavioral } \\
\text { predictor }\end{array}$} & V6 & - Length of time to smoke the favorite cigarette brand & Continuous & {$[16,70]$} \\
\hline & V7-V12 & $\begin{array}{l}\text { - Whether to buy cigarettes in department store, supermarket, convenience } \\
\text { store, grocery, tobacco dealer shop or hotel/Restaurant/public entertainment } \\
\text { places }\end{array}$ & Binary & $\{0,1\}$ \\
\hline & V13-V23 & $\begin{array}{l}\text { - Importance level of individual habit, taste, product awareness, package, } \\
\text { cigarette shred, tar content, family/friends' influence, flavor, salesman rec- } \\
\text { ommendation, product promotion, advertisement in purchase }\end{array}$ & Ordinal & $\{1,2,3,4\}$ \\
\hline & V24-V30 & $\begin{array}{l}\text { - Importance level of price, taste, cigarette shred, individual habit, reputa- } \\
\text { tion of production place, tar content, flavor on brand choice }\end{array}$ & Ordinal & $\{1,2,3,4\}$ \\
\hline & $\mathrm{V} 31-\mathrm{V} 40$ & $\begin{array}{l}\text { - Importance level of income change, taste change, product quality, flavor, } \\
\text { price, friend/family recommendation, brand popularity, convenience of pur- } \\
\text { chase, attempt of new brand, product promotion in brand switch }\end{array}$ & Ordinal & $\{1,2,3,4\}$ \\
\hline \multirow{3}{*}{$\begin{array}{l}\text { Perception } \\
\text { predictor }\end{array}$} & V41 & - Price sensitivity & Ordinal & $\{1,2,3\}$ \\
\hline & V42-V47 & $\begin{array}{l}\text { - Importance level of cigarette shred, cigarette ash, feel, package, taste, tar } \\
\text { content in product evaluation }\end{array}$ & Ordinal & $\{1,2,3,4\}$ \\
\hline & V48-V57 & $\begin{array}{l}\text { - Acceptability level of quantity discount, gifts, free samples, TV advertise- } \\
\text { ment, newspaper advertisement, roadside advertisement, poster, SMS lot- } \\
\text { tery, empty boxes for prize, arts performance in promotion }\end{array}$ & Ordinal & $\{1,2,3,4,5,6\}$ \\
\hline
\end{tabular}

